\title{
Cultivo de Kalanchoe blossfeldiana em diferentes substratos contendo resíduos de
}

\section{erva-mate}

\author{
Kalanchoe blossfeldiana cultivation on different substrates containing yerba mate residues
}

Cultivo de Kalanchoe blossfeldiana sobre diferentes sustratos que contienen residuos de yerba mate

Recebido: 05/04/2021 | Revisado: 14/04/2021 | Aceito: 16/04/2021 | Publicado: 01/05/2021

Daniela Machado Monteiro
ORCID: https://orcid.org/0000-0002-9396-8453
Enstituto de Desenvolvimento Educacional de Passo Fundo, Brasil
E-mail: machadodaniela62@ @mail.com
Leonita Beatriz Girardi
ORCID: https://orcid.org/0000-0003-2035-2866
E-mail: lbgirardi @ hotmail.com
Janine Farias Menegaes
Instituto de Desenvolvimento Educacional de Paso Fundo, Brasil
ORCID: https://orcid.org/000-0001-6053-4221
Universidade Federal de Santa Maria, Brasil
E-mail: janine_rs@ hotmail.com
Cristian José Steffens
ORCID: https://orcid.org/0000-0002-0459-4753
E-mail: cristiansteffens14@gmail.com
Instituto de Desenvolvimento Educacional de Passo Fundo, Brasil
Jackson Vinícius Rodrigues Pereira
ORCID: https://orcid.org/0000-0003-3919-4044
E-mail: jacksonvrp@hotmail.com
Tarik Ian Reinehr
Instituto de Desenvolvimento Educacional de Paso Fundo, Brasil
ORCID: https://orcid.org/0000-0001-9363-8131
Universidade de Passo Fundo, Brasil
E-mail: tarik-ian@hotmail.com

\section{Resumo}

Em busca de uma agricultura de baixo impacto ambiental, o objetivo com este trabalho foi avaliar o cultivo de Kalanchoe blossfeldiana Poelln. (calandivas) cultivar Amora, submetidas a diferentes substratos contendo na sua composição resíduos de erva-mate. O experimento foi conduzido em delineamento inteiramente casualizado, com cinco tratamentos compostos pelas diferentes misturas do substrato comercial e o resíduo de erva-mate compostada, obtendo as composições: S1: 100\% substrato comercial (SC); S2: 50\% SC + 50\% resíduo de erva-mate (REM); S3: 25\% SC + 75\% REM; S4: 75\% SC + 25\% REM e S5: 100\% REM, com cinco repetições, cada unidade experimental foi constituída por um vaso de planta. Analisou-se as variáveis quanto à altura das plantas e comprimento radicular, diâmetro das inflorescências, número de flores e folhas e massa fresca radicular. Observou-se que o desenvolvimento das plantas de calandivas foi similar com as diferentes combinações contendo o substrato comercial e o resíduo de erva-mate. No entanto, o substrato contendo apenas com os resíduos de erva-mate não é recomendado para esse cultivo. Por fim, recomenda-se para o cultivo de calandivas em vasos o uso do substrato composto de $50 \%$ substrato comercial $+50 \%$ resíduo de erva-mate $(\mathrm{S} 2)$.

Palavras-chave: Flor-de-vaso; Reuso de substâncias orgânicas; Produção local de flores.

\begin{abstract}
In search of a low environmental impact agriculture, the objective of this work was to evaluate the cultivation of Kalanchoe blossfeldiana Poelln. (calandiva) cultivate Amora, submitted to different substrates containing yerba mate residues in its composition. The experiment was carried out in a completely randomized design, with five treatments composed by the different mixtures of the commercial substrate and the composted yerba mate residue, obtaining the compositions: S1: $100 \%$ commercial substrate (SC); S2: $50 \%$ SC + 50\% yerba mate residue (REM); S3: $25 \%$ SC + 75\% REM; S4: 75\% SC + 25\% REM and S5: 100\% REM, with five replications, each experimental unit consisted of a plant pot. The variables were analyzed in terms of plant height and root length, inflorescence diameter, number of flowers and leaves and fresh root mass. It was observed that the development of calandiva plants was similar with the different combinations containing the commercial substrate and the residue of yerba mate. However, the substrate containing only the residues of yerba mate is not recommended for this cultivation. Finally, it is recommended for the
\end{abstract}


cultivation of calandiva in pots the use of the substrate composed of 50\% commercial substrate $+50 \%$ yerba mate residue (S2).

Keywords: Potted flower; Reuse of organic substances; Local flower production.

\section{Resumen}

En busca de una agricultura de bajo impacto ambiental, el objetivo de este trabajo fue evaluar el cultivo de Kalanchoe blossfeldiana Poelln. (calandiva) cultivan Amora, sometida a diferentes sustratos que contienen residuos de yerba mate en su composición. El experimento se realizó en un diseño completamente al azar, con cinco tratamientos compuestos por las diferentes mezclas del sustrato comercial y el residuo de yerba mate compostado, obteniendo las composiciones: S1: sustrato $100 \%$ comercial (SC); S2: $50 \%$ de SC + 50\% de residuo de yerba mate (REM); S3: $25 \%$ SC + 75\% REM; S4: 75\% SC + 25\% REM y S5: $100 \%$ REM, con cinco repeticiones, cada unidad experimental consistió en una maceta. Las variables se analizaron en términos de altura de planta y longitud de raíz, diámetro de inflorescencia, número de flores y hojas y masa de raíz fresca. Se observó que el desarrollo de las plantas de calandiva fue similar con las diferentes combinaciones que contienen el sustrato comercial y el residuo de yerba mate. Sin embargo, el sustrato que contiene solo los residuos de yerba mate no se recomienda para este cultivo. Finalmente, se recomienda para el cultivo de calandiva en macetas el uso del sustrato compuesto por $50 \%$ de sustrato comercial + $50 \%$ de residuo de yerba mate (S2).

Palabras clave: Flor en maceta; Reutilización de sustancias orgánicas; Producción local de flores.

\section{Introdução}

A produção comercial de espécies ornamentais no Rio Grande do Sul (RS) está em constante crescimento, apesar de inúmeras restrições entre as quais, condições climáticas, além de pouco incentivo governamental e falta de assistência técnica especializada. O RS é autossuficiente apenas em forrações ornamentais (caixarias), para todas as demais espécies é necessário aporte de regiões produtoras, sendo a região Sudeste a que mais abastece o Estado (Kämpf \& Daudt, 1999; Menegaes et al., 2015).

Todavia, há uma grande demanda no Estado por flores envasadas, entre elas destaca-se a calandiva ou flor-da-fortuna (Kalanchoe blossfeldiana Poelln.), em função da exuberância de suas flores e pela importância simbólica do nome da planta, o qual é atribuído a facilidade de propagação e espessura das folhas. De cultivo anual a espécie aprecia frio e apresenta grande variedade de coloração da inflorescência pelos diferentes tons de rosa, vermelho, branco e amarelo (Kämpf, 2000; Lorenzi, 2013).

O cultivo em flores e plantas ornamentais em vaso é o resultado de vários fatores, entre eles a escolha do substrato e dos seus constituintes é de suma importância para a qualidade do produto final. Uma vez que, o substrato visa proporcionar estrutura física, química e biológica ideal para o desenvolvimento e fixação radicular, bem como o desenvolvimento pleno da planta, além de maximizar o uso racional da água e de nutrientes (Kämpf, Takane \& Siqueira, 2006; Menegaes et al., 2020).

Os constituintes da composição do substrato devem ser adaptados ao local mais próximo da área de cultivo e se possível de material a partir de reuso, conforme a disponibilidade na região e, também, de baixo custo. Atualmente, há uma grande variedade de compostos para a formulação dos substratos, como areia, solo, casca de arroz carbonizada, casca de pinus, resíduos de compostagem, entre outros (Fermino, 2014; Guimarães \& Feitosa, 2015).

A compostagem de resíduos de subprodutos e matérias orgânicos, é um método que vem auxiliar a crescente demanda pela obtenção de fertilizantes orgânicos. Também, possuem propriedades biológicas adequadas para o seu uso como substratos, especialmente em sistemas de produção sustentáveis, causando menores impactos ambientais e sociais de aspectos negativos, consequentemente reduz a utilização de insumos externos (Takane et al., 2013; March \& Gonçalves, 2020).

Entre os resíduos de origem orgânica na região Sul do país, encontra-se os resíduos de erva-mate (Ilex paraguaniensis A. St-Hill.), os quais são oriundos do hábito de tomar chimarrão diariamente. Contudo, a erva-mate apresenta saponinas e outros compostos que necessitam passar por compostagem ou vermicompostagem antes do uso como constituinte de substrato (Kämpf, 2000). 
Assim, em busca de uma agricultura de baixo impacto ambiental, o objetivo do presente trabalho foi avaliar o cultivo de calandivas submetidas a diferentes substratos contendo na sua composição resíduos de erva-mate.

\section{Metodologia}

O experimento foi realizado nos meses de abril a agosto de 2020, em casa de vegetação localizada na cidade de Passo

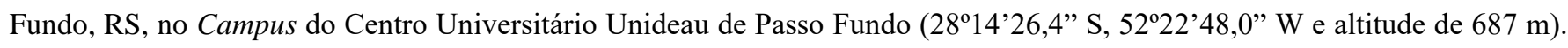
O clima da região se caracteriza pela Zona Climática fundamental temperada (C) apresentando o clima tipicamente fundamental úmido (f) e variedade específica subtropical (Cfa).

O experimento foi conduzido em delineamento inteiramente casualizado, com cinco tratamentos compostos pelas diferentes misturas do substrato comercial Dubom ${ }^{\circledR}$ e o resíduo de erva-mate compostada, obtendo as composições: S1: 100\% substrato comercial; S2: $50 \%$ substrato comercial $+50 \%$ resíduo de erva-mate; S3: $25 \%$ substrato comercial + 75\% resíduo de erva-mate; S4: $75 \%$ substrato comercial $+25 \%$ resíduo de erva-mate e S5: 100\% resíduo de erva-mate, com cinco repetições, cada unidade experimental foi constituída por um vaso de planta. Após a elaboração os substratos foram enviados para análise química conforme a Tabela 1. O resíduo de erva-mate após o uso em forma de chimarrão foi coletado, compostado e seco por 28 dias.

Tabela 1 - Laudo da análise dos substratos*.

\begin{tabular}{|c|c|c|c|c|c|c|c|c|}
\hline \multirow[t]{2}{*}{ Substratos } & $\begin{array}{c}\text { Umidade } \\
65^{\circ} \mathrm{C}\end{array}$ & $\mathbf{N}^{1}$ & $\mathbf{P}_{2} \mathrm{O}_{5}{ }^{1}$ & $\mathrm{~K}_{2} \mathrm{O}^{2}$ & C org. & \multirow{2}{*}{$\begin{array}{c}\text { pH em } \\
\mathrm{H}_{2} \mathrm{O}\end{array}$} & \multirow{2}{*}{$\begin{array}{c}\text { Relação } \\
\text { C/N }\end{array}$} & \multirow{2}{*}{$\begin{array}{c}\text { C.E. } \\
\left(\mathrm{mS} \mathrm{cm}^{-1}\right)\end{array}$} \\
\hline & \multicolumn{5}{|c|}{ - } & & & \\
\hline Substrato 1 & 49,50 & 0,49 & 0,70 & $<0,25$ & 11,10 & 6,85 & 22,65 & 1,27 \\
\hline Substrato 2 & 19,27 & 3,22 & 0,90 & 0,81 & 26,47 & 7,77 & 8,22 & 3,77 \\
\hline Substrato 3 & 17,99 & 2,66 & 0,72 & 0,82 & 31,49 & 7,99 & 11,84 & 3,44 \\
\hline Substrato 4 & 39,11 & 1,54 & 0,73 & $<0,25$ & 17,40 & 7,63 & 11,30 & 1,67 \\
\hline Substrato 5 & 59,94 & 2,73 & 0,16 & 0,80 & 16,79 & 8,95 & 6,15 & 3,19 \\
\hline
\end{tabular}

* Métodos oficiais do Mistério da Agricultura, Pecuária e Abastecimento pelas Instruções Normativas n. ${ }^{\circ} 24 / 2007$ e n. ${ }^{\circ} 37 / 2017 .{ }^{1}$ teor total; ${ }^{2}$ solúvel em água. Fonte: Autores.

A cultivar de calandiva utilizada foi a "Amora" (coloração da inflorescência vermelho) e as mudas, foram adquiridas da empresa Úrsula, com altura de $5 \mathrm{~cm}$. O plantio das mudas ocorreu nos vasos plásticos no $9(7,4 \mathrm{~cm}$ de altura e $10 \mathrm{~cm}$ de largura, com o volume total de $0,45 \mathrm{~L}$ ), utilizado para o cultivo comercial desta cultura, contendo os substratos supracitados. Para a irrigação das plantas utilizou-se regador, sendo a irrigação efetuada uma vez por semana de acordo as necessitas da espécie.

Foram analisadas as variáveis de desenvolvimento quanto à altura das plantas e comprimento radicular ambos com uso de régua milimetrada, ao diâmetro das inflorescências com o uso de paquímetro digital, ao número de flores por inflorescência e de folhas por contagem manual, massa fresca radicular com uso de balança digital.

Os dados foram submetidos a análise de variância e havendo significância para o teste $\mathrm{F}$, os tratamentos foram comparados pelo teste de variância de Tukey $(\mathrm{p}<0,05)$, com auxílio da planilha eletrônica Office Excel e do programa estatístico SISVAR (Ferreira, 2014). 


\section{Resultados e Discussão}

As alturas das plantas de calandiva da cultivar Amora apresentaram diferença estatística para os substratos testados, onde o tratamento S1 (100\% substrato comercial) obteve melhor resultado em relação aos demais (Tabela 2). Seguidos dos tratamentos S2 (50\% substrato comercial + 50\% resíduo de erva-mate) e S4 (75\% substrato comercial + 25\% resíduo de ervamate), os quais demonstraram desenvolvimento uniforme e harmônico de plantas.

Tabela 2 - Avaliação de estatura de plantas de calandivas (Kalanchoe blossfeldiana Poelln.) cv. Amora cultivadas em diferentes composições de substratos.

\begin{tabular}{|c|c|c|c|c|}
\hline Substratos & Altura (cm) & $\begin{array}{c}\text { Número de folhas } \\
\text { (unidade) }\end{array}$ & $\begin{array}{c}\text { Emergência do receptáculo } \\
\text { floral (SAP) }\end{array}$ & $\begin{array}{l}\text { Diâmetro da inflorescência } \\
(\mathbf{m m})\end{array}$ \\
\hline Substrato 1 & $27,15 a^{*}$ & $42,9^{\mathrm{ns}}$ & $6 a^{*}$ & $15,00 \mathrm{c}$ \\
\hline Substrato 2 & $26,49 \mathrm{~b}$ & 42,5 & $4 \mathrm{c}$ & $19,41 a^{*}$ \\
\hline Substrato 3 & $22,38 \mathrm{~d}$ & 40,0 & $5 \mathrm{~b}$ & $16,53 \mathrm{~b}$ \\
\hline Substrato 4 & $26,32 \mathrm{~b}$ & 38,6 & $5 \mathrm{~b}$ & $16,84 \mathrm{~b}$ \\
\hline Substrato 5 & $23,38 \mathrm{c}$ & 40,7 & $3 \mathrm{~d}$ & $16,67 \mathrm{~b}$ \\
\hline Média & 25,14 & 40,9 & 4,6 & 16,89 \\
\hline \multirow[t]{2}{*}{$\mathrm{CV}$} & $1,08 \%$ & $5,97 \%$ & & \\
\hline & $\begin{array}{l}\text { Número de flores } \\
\text { (unidade) }\end{array}$ & $\begin{array}{l}\text { Comprimento radicular } \\
\qquad(\mathrm{cm})\end{array}$ & Massa fresca radicular (g) & \\
\hline Substrato 1 & $16 \mathrm{c}$ & $9,14 \mathrm{a}^{*}$ & $7,29 a^{*}$ & \\
\hline Substrato 2 & $36 \mathrm{a}^{*}$ & $9,34 \mathrm{a}$ & $3,58 \mathrm{c}$ & \\
\hline Substrato 3 & $25 \mathrm{~b}$ & $3,82 \mathrm{~d}$ & $2,63 \mathrm{e}$ & \\
\hline Substrato 4 & $28 \mathrm{~b}$ & $6,92 \mathrm{~b}$ & $4,82 \mathrm{~b}$ & \\
\hline Substrato 5 & $18 \mathrm{c}$ & $2,47 \mathrm{e}$ & $3,23 \mathrm{~d}$ & \\
\hline Média & 24,6 & 6,34 & 4,31 & \\
\hline $\mathrm{CV}$ & & $3,89 \%$ & $2,99 \%$ & \\
\hline
\end{tabular}

*interação significativa e ${ }^{\mathrm{ns}}$ interação não significativa dos tratamentos. As médias não seguidas pelas mesmas letras diferem estaticamente pelo teste de Tukey $(\mathrm{p}<0,05)$. CV: Coeficiente de variação. SAP: semanas após o plantio.

Quanto ao número de folhas por plantas, sob o desenvolvimento em diferentes substratos não diferiram estatisticamente entre si, mantendo a média de aproximadamente 40,9 folhas por planta. De acordo com Souza et al. (2010), observaram para o cultivo de kalanchoe utilizando substratos alternativos, que o maior número médio de folhas foi encontrado nas plantas cultivadas com o composto contendo casca de arroz carbonizada (CAC) + esterco avícola (EA), apresenta espaço de aeração ideal para o desenvolvimento da planta, diferenciando-se dos substratos CAC e cinza de casca de arroz (CZ).

A emergência do receptáculo floral (Tabela 2 e Figura 1) ocorreu primeiramente no tratamento S5 (100\% resíduo de erva-mate) apresentando o receptáculo floral bem desenvolvido três semanas após do plantio (SAP) das mudas. Na semana seguinte, as plantas cultivadas no tratamento S2, seguidos dos demais tratamentos sendo tratamento S3 (25\% substrato comercial $+75 \%$ resíduo de erva-mate) e S4. Estes tratamentos pertence a mistura do resíduo da erva-mate junto ao substrato comercial. 
Figura 1 - Emergências dos receptáculos florais das calandivas (Kalanchoe blossfeldiana Poelln.) cv. Amora cultivadas em diferentes composições de substratos.
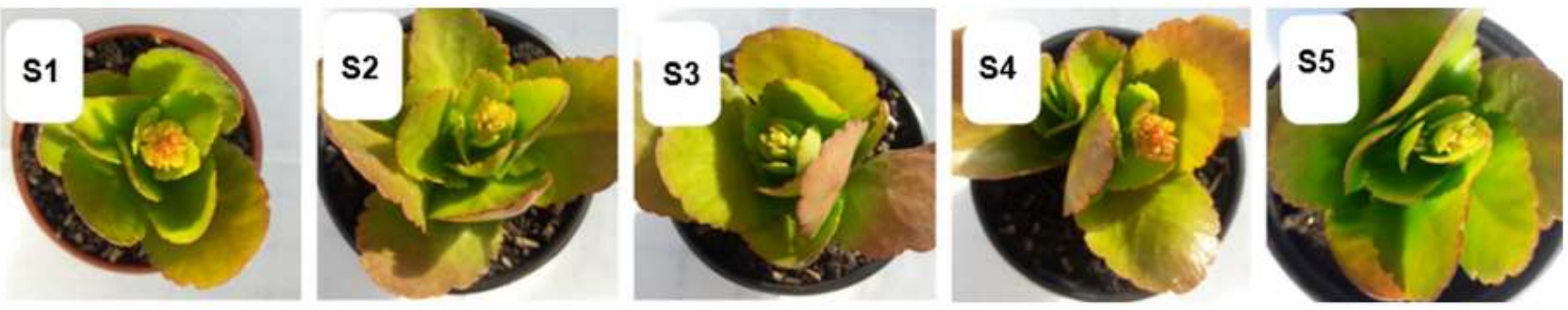

S1: $100 \%$ substrato comercial; S2: $50 \%$ substrato comercial $+50 \%$ resíduo de erva-mate; S3: $25 \%$ substrato comercial $+75 \%$ resíduo de erva-mate; S4: $75 \%$ substrato comercial + 25\% resíduo de erva-mate S5: 100\% resíduo de erva-mate. Fonte: Autores.

Quanto ao tratamento S1 observou-se emergência tardia do receptáculo floral, levando até seis SAP das mudas para emergência do receptáculo. Pontua-se que os substratos contendo resíduos de erva-mate + substrato comercial contribuíram para o desenvolvimento acelerado do receptáculo floral. Para Terra et al. (2011), em que trabalharam com o cultivo de crisântemos (Dendranthema grandiflora Tzvelev.) em vasos sob diferentes substratos que para comprimento de raiz e quanto a precocidade da emissão dos botões florais, obteve-se resultados mais acentuados para o substrato comercial, assim, demostrouse superior quanto a valores de nutrientes, níveis de fertilidade do solo desde o período inicial de cultivo, estes fatores podem influenciar assim na emissão precoce dos botões florais além de um maior número destes.

Os diâmetros das inflorescências foram diferentes para todos tratamentos, sendo o maior com a utilização do tratamento S2 (50\% substrato comercial $+50 \%$ resíduo de erva-mate), em que possivelmente a mistura $50 \%$ de cada constituinte favoreceu melhor desenvolvimento, favorecendo a maior emissão de número de flores (Tabela 2 e Figura 2A).

Em experimento com gérberas (Gerbera jamesonii L.) de vasos conduzidas em diferentes substratos, Ludwig et al. (2010), descreveu que as características de qualidade estética podem variar para as diferentes espécies consideradas planta ornamental e para flor envasada, portanto é importante considerar a formação da folhagem e da inflorescência.

Figura 2 - Abertura dos botões florais das calandivas (Kalanchoe blossfeldiana Poelln.) cv. Amora (A) e coesão dos substratos (B), sob diferentes composições de substratos.

A

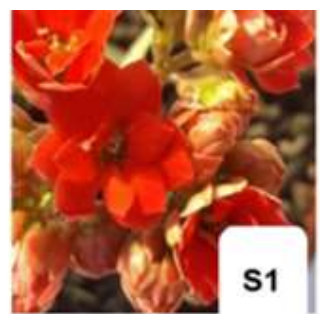

B

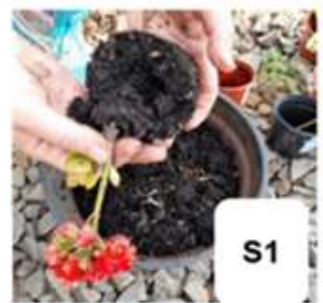

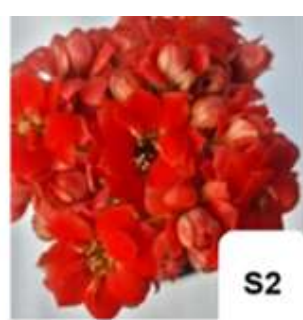

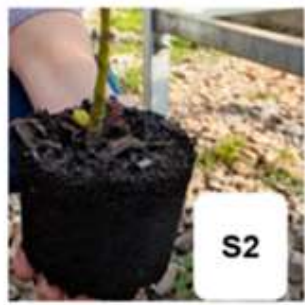

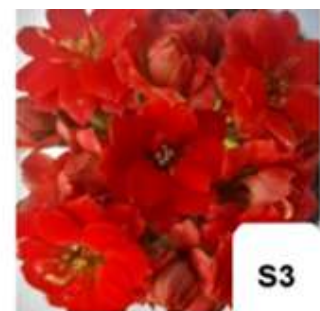
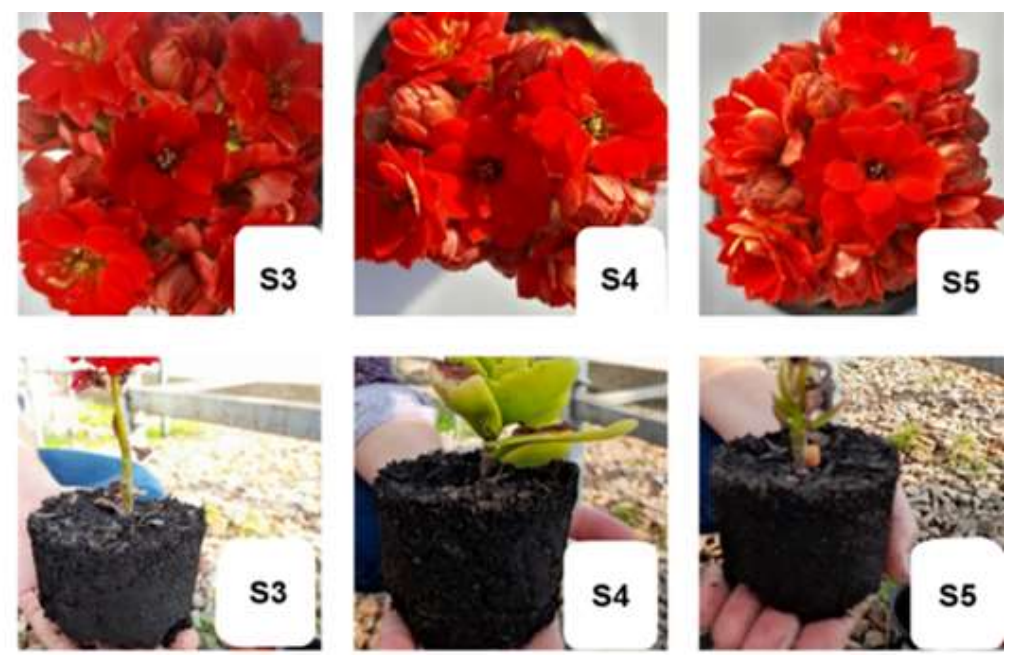

S1: $100 \%$ substrato comercial; S2: $50 \%$ substrato comercial $+50 \%$ resíduo de erva-mate; S3: $25 \%$ substrato comercial $+75 \%$ resíduo de erva-mate; S4: 75\% substrato comercial + 25\% resíduo de erva-mate S5: 100\% resíduo de erva-mate. Fonte: Autores.] 
Para Souza et al. (2010) em seu experimento com calandiva enfatizou que, o maior número de inflorescências por planta foi observado nos substratos contendp cinza de casca de arroz (CZ) e CZ + esterco bovino (EB). Kämpf, Takane \& Siqueira (2006) observaram que este aumento pode estar relacionado com a capacidade de retenção de água, uma vez que estes apresentaram valores satisfatórios para este aspecto físico. Quanto ao número de flores por planta, o substrato $\mathrm{CZ}+\mathrm{EB}$ apresentou o maior valor, em razão de apresentar condições ideais de retenção de água. Para Lessa et al. (2009) e Souza et al. (2010), para espécies de calandiva, apenas uma dessas variáveis não pode ser levada em consideração por si só para determinar seu aspecto ornamental, mas sim o conjunto destas é que atenderão o padrão comercial exigido.

Para o comprimento radicular observou-se que os tratamentos diferiram-se entre si, quanto ao desenvolvimento do sistema radicular, especialmente nos tratamentos S2 e S1. Quanto a massa fresca das raízes, todos os tratamentos diferiram-se entre si, sendo que a massa maior encontrada foi para no tratamento S1 (100\% substrato comercial). Silva et al. (2019) descreveu em trabalho de pesquisa do desenvolvimento de mudas de sempre-viva (Helichrysum bracteatum Andrews.), submetidos a diferentes substratos, que para o comprimento de raízes e comprimento da parte área, observou-se que o substrato do tratamento $\mathrm{S} 4$, sendo este composto de vermiculita de textura fina, apresentou melhores resultados perante estas variáveis, comparado aos outros tratamentos, no entanto, não diferiu estatisticamente dos substratos.

O substrato que melhor apresentou coesão (Figura 2B), foi o tratamento S4, na sequência os tratamentos S3 e S5, foram os substratos que se apresentaram de forma negativa perante os demais, pois os mesmos apresentaram pouca coesão de torrão e maior retenção de umidade que os demais substratos. Para os tratamentos S2 e S1 obteve-se bons aspecto de coesão de torrão e pouca retenção de umidade na hora da retirada dos vasos, demonstrando semelhança entre si.

A coesão do substrato contribui para a retirada da planta quando for realizada o replantio desta em outro local, não havendo perda gradativa da estabilidade da planta, além das raízes, não gerando estresse para planta. Para Menegaes et al. (2017), os parâmetros de comprimento radicular e de massa fresca radicular favorecem a estabilidade de coesão favorecendo um bom desenvolvimento na relação parte aérea e parte radicular.

\section{Conclusão}

Conclui-se que o substrato composto apenas com os resíduos de erva-mate afetou negativamente os aspectos qualitativos das plantas de calandivas. Todavia, plantas cultivadas com as composições de substratos contendo até 50\% deste resíduo apresentaram maior número de flores e maior diâmetro da inflorescência. Assim, recomenda-se para o cultivo de calandivas em vasos o uso do substrato composto de 50\% substrato comercial + 50\% resíduo de erva-mate (S2).

\section{Referências}

Fermino, M. H. (2014). Substratos: composição, características e métodos de análise. Agrolivros.

Ferreira, D. F. (2014). Sisvar: A guide for is bootstrap procedures in multiple comparisons. Ciência e Agrotecnologia, 38(2), 109-112.

Guimarães, M. A., \& Feitosa, F. R. C. (2015). Implantação de hortas: aspectos a serem considerações. PRONTOGRAF.

Kämpf, A. N. (2000). Produção comercial de plantas ornamentais. Agropecuária, 2000. 254p.

Kämpf, A. N., \& Daudt, R. S. (1999). Diagnóstico da floricultura no Rio Grande do Sul. Revista Ciência Rural, 29(3), 561-563.

Kämpf, A. N., Takane, R., \& Siqueira, P.T.V. (2006). Floricultura - técnicas de preparo de substratos. Tecnologia Fácil.

Lessa, M. A., Paiva, P. D. O., Alves, C. M. L., \& Resende, M. L. (2009). Application of different fertilizers in substrate for Kalanchoe luciae Raym-Hamet cultivation. Ciência e Agrotecnologia, 33(4), 950-955.

Lorenzi, H. (2013). Plantas para jarim no Brasil - herbáceas, arbustivas e trepadeiras. Instituto Plantarum.

Ludwig, F., Guerrero, A. C., Fernandes, D. M., \& Villas Boas, R, L. (2010). Análise de crescimento de gérbera de vaso conduzida em diferentes substratos. Horticultura Brasileira, 28(1), 70-74. 
Research, Society and Development, v. 10, n. 5, e12710514683, 2021

(CC BY 4.0) | ISSN 2525-3409 | DOI: http://dx.doi.org/10.33448/rsd-v10i5.14683

Marchi, C. M. D., \& Gonçalves, I. O. (2020). Compostagem: a importância da reutilização dos resíduos orgânicos para a sustentabilidade de uma instituição de ensino superior. REMOA, 19(1), 1-25.

Menegaes, J. F., Backes, F. A. A. L., Bellé, R. A., \& Backes, R. L. (2015). Diagnóstico do mercado varejista de flores de Santa Maria, RS. Ornamental Horticulture, 21(3), 291-298.

Menegaes, J. F., Fiorin, T. T., \& Rodrigues, A. M. (2020). Emergência de plântulas e produção de mudas de couve-flor em diferentes substratos e regime de irrigação. Acta Iguazu, 9(4), 109-117.

Menegaes, J. F., Zago, A. P., Bellé, R. A. \& Backes, F. A. A. L. (2017). Enraizamento de estacas de forrações ornamentais em diferentes concentrações de ácido indolbutírico. Nativa, 5(5), 311-315.

Silva. J. N., Freire, A. S., Rodrigues, M. H. S., Silva, M. A., Silva, L. F. Desenvolvimento inicial de mudas de Helichrysum bracteatum submetidas a diferentes substratos. Meio Ambiente, v. 1, n. 3, p. 09-15, 2019.

Souza, A. R. C., Peiter, M. X., Robaina, A. D., Sores, F. C., Parizi, A. R. C., \& Ferraz, R. C. (2010). Consumo hídrico e desempenho de Kalanchoe cultivado em substratos alternativos. Ciência Rural, 40(3), 534-540.

Takane, R. J., Yanagisawa, S. S., \& Góis, E. A. (2013). Técnicas em substratos para a floricultura. Expressão Gráfica.

Terra, S. B., Ferreira, A. A. F., Peil, R. M. N., Stumpf, E. R. T., Beckmann-Cavalcante, M. Z., \& Cavalcante, I. H. L. (2011). Alternative substrates for growth and production of potted chrysanthemum (cv. Funny). Acta Scientiarum - Agronomy, 33(3), 465-471. 